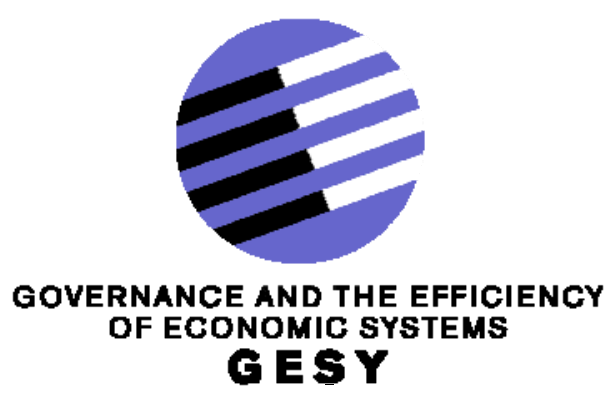

Discussion Paper No. 377

Optimal Use of Rewards as Commitment Device When Bidding is Costly

Luke Hu*

${ }^{*}$ Humboldt University at Berlin

Februar 2012

Financial support from the Deutsche Forschungsgemeinschaft through SFB/TR 15 is gratefully acknowledged. 


\title{
Optimal Use of Rewards as Commitment Device When Bidding is Costly ${ }^{\text {th }}$
}

\author{
Luke $\mathrm{Hu}$ \\ Institute of Economic Theory I, Humboldt University at Berlin \\ Spandauer Str. 1, 10178 Berlin, Germany
}

\begin{abstract}
This paper considers procurement auctions with costly bidding when the auctioneer is unable to commit himself to restrict the number of bidders. The auctioneer can, however, offer a financial reward to be paid to every short-listed bidders as an indirect commitment device. Rewards for short-listed bidders are costly. Nevertheless, it is generally optimal for the procurer to credibly implement the same restriction of the number of bidders that is optimal under full commitment.
\end{abstract}

Keywords: Procurement, auctions, industrial organization, mechanism design. JEL: D21, D43, D44, D45.

\section{Introduction}

A common feature of many procurement auctions is that bidding is costly. Contractors must assess the job to be done which often requires extensive explorations and detailed bid proposals. In the presence of such bidding costs, bidder participation and bids depend significantly on the expected number of competitors in such a way that more competition is disadvantageous to the auctioneer (see Lang and Rosenthal, 1991; Tan, 1992). Therefore, the auctioneer would like to convince bidders that he invites only a limited number of bidders.

If the auctioneer is a major non-profit organization or government agency, the auctioneer can commit himself to invite only a limited number of bidders to participate in the auction. ${ }^{1}$ However, in many applications, such commitment is not feasible. For example, if a family wants to hire a contractor to renovate their house, they can always invite yet another contractor; they may want contractors to believe that they restricts bidding, but they will benefit from breaching that trust. In that case, bidders consider any announcement concerning the size of the short-list of bidders as "cheap talk" and make their own rational predictions concerning the number of bidders before they bid.

\footnotetext{
I thank Elmar Wolfstetter for advice and suggestions. Research support by the Deutsche Forschungsgemeinschaft (DFG), SFB Transregio 15, "Governance and Efficiency of Economic Systems" is gratefully acknowledged.

Email address: hu_luke@yahoo.com (Luke $\mathrm{Hu}$ )

${ }^{1}$ For example, the World Bank is bound by its procurement rules to short-list six bidders, if feasible, and to publish the short-list of bidders when they are invited to make a bid.
} 
On this background, the present paper explores the use of rewards for shortlisted bidders as an indirect commitment device. There, the procurer offers to pay a financial reward to every bidder who is invited to bid. Such a reward makes it profitable to the procurer invite only a limited number of bidders. Bidders then use the information conveyed by that reward to predict how many bidders are actually invited to bid.

There is a small literature on using rewards for bidder when bidding is costly. Gal, Landsberger and Nemirovski (2007), Kaplan and Sela (2006), and Fan and Wolfstetter (2008) explore whether reimbursing bidding costs may profitably change the composition of participating bidders. However, unlike in this literature, the present paper characterizes the rewards necessary to back up an announced size of the short-list, and solve the optimal reward and associated size of the short-list. Since rewards are costly, there is a trade-off between cost and size of the short-list. Nevertheless, we find that it is optimal to implement exactly the same size of the short-list as under full commitment.

\section{Model}

We consider a variation of the well-known "contractor's game" by Lang and Rosenthal (1991). There, a procurer faces a large number of potential contractors $n \geq 3$ and invites a subset of contractors to bid in a first-price (reverse) auction. Performing the job is costly and so is bidding. A bid is an offer to perform the job in exchange for a requested price called bid.

Bidders have two kinds of common value costs: the uncertain performance cost, $c$, and the commonly known bidding cost, $e \in(0,1)$. The performance cost is drawn from the continuous probability distribution $F:[0,1-e] \rightarrow[0,1]$ with twice continuously differentiable p.d.f. $f$.

The game is as follows:

1. Nature draws the common value performance cost $c$ from the c.d.f. $F$ which is observed by all contractors but not by the auctioneer.

2 . The procurer announces the size of the short-list and invites $m \leq n$ contractors to bid in a first-price (reverse) auction with reserve price $R=1$, and pays every short-listed bidder a financial reward, $r$. Bidders cannot observe how many bidders are invited to bid.

3. Bidders predict how many contractors are invited to bid, and simultaneously decide whether to bid and if they bid how much to bid.

4. The procurer selects the lowest bidder as winner (provided that bid does not exceed the reserve price $R$ ), and the winner performs the job.

Without loss of generality, we only consider combinations of $m$ and $r$ for which it is in the best interest of the procurer to invite exactly the announced number of $m$ bidders. ${ }^{2}$

For each given combination $(m, r)$ the bidding subgame is exactly the "contractor's game" by Lang and Rosenthal (1991). That game has a unique equilibrium. There, each of the $m$ bidders makes a bid with probability $q$ and, if he

\footnotetext{
${ }^{2}$ For simplicity, one may invoke that bidders do not participate in the auction if they predict that the procurer invites more than the announced number of bidders. This avoids glutted notation that would be necessary if one allowed for discrepancies between the announced size of the short-list and bidders' prediction of it.
} 
bids, draws his bid at random from the c.d.f. of bids $B:[c+e, 1] \rightarrow[0,1]$ :

$$
q(m, c)=1-\left(\frac{e}{1-c}\right)^{\frac{1}{m-1}}, \quad B(b ; m, c)=\frac{1-\left(\frac{e}{b-c}\right)^{\frac{1}{m-1}}}{1-\left(\frac{e}{1-c}\right)^{\frac{1}{m-1}}} .
$$

Obviously the equilibrium $(q, B)$ is independent of the reward for bidding; that reward only matters to credibly back the announced number of invited bidders.

An implication of this result is that the probability distribution of the equilibrium price is stochastically increasing in the number of bidders $m$, in the sense of first-order stochastic dominance (Lang and Rosenthal, 1991, see Theorem 2). Therefore, if the procurer can commit to restrict the number of bidders, he should short-list exactly $m=2$ bidders.

\section{Rewards for short-listed bidders as commitment device}

We now assume that the procurer uses the reward, $r$, to credibly implement an announced short-list of $m$ bidders.

For this purpose, suppose the procurer has announced $(m, r)$, bidders believe that he will invite $m$ bidders, but the procurer deviates and invites $a$ bidders to participate in the auction. Denote the associated deviation payoff of the procurer by $\Pi(m, r, a)$, and one finds (the detailed proof is in the Appendix):

Lemma 1. The deviation payoff of the procurer is equal to

$$
\Pi(m, r, a)=\int_{0}^{1-e}\left(1-e-c-\int_{c+e}^{1}(1-q(m, c) B(b ; m, c))^{a} d b\right) d F(c)-a r .
$$

A short-list of $m$ together with a reward for bidding, $r$, is "credible" if and only if the procurer can neither benefit from inviting more bidders (upward deviation) nor from inviting fewer bidders (downward deviation). For convenience define $\pi(m, r):=\Pi(m, r, m)$. Then, upward deviations are prevented if and only if

$$
\pi(m, r) \geq \Pi(m, r, a), \text { for all } a>m, a, m \in N .
$$

Similarly, downwards deviations are prevented if and only if $\pi(m, r) \geq \Pi(m, r, a)$, for all $a<m, a, m \in N$.

Lemma 2. The smallest reward that deters upward deviations, denoted by $r^{*}(m)$, is equal to

$$
r^{*}(m)=\int_{0}^{1-e} \int_{c+e}^{1}\left((1-q(m, c) B(b ; m, c))^{m} q(m, c) B(b ; m, c)\right) d b d F(c) .
$$

Proof. Suppose the procurer has announced the mechanism $\left(m, r^{*}(m)\right)$ and bidders believe that he invites exactly $m$ bidders to participate in the auction. We prove by induction that $r^{*}(m)$ is the smallest reward that assures that the procurer does not benefit from inviting more than $m$ bidders.

1) We show that $r^{*}(m)=\inf \{r \mid \pi(m, r) \geq \Pi(m, r, m+1)\}$. The reward $r^{*}(m)$ deters the procurer from inviting one more bidder, and it is the smallest 
reward that achieves this goal. This follows from

$$
\begin{aligned}
\pi\left(m, r^{*}\right)= & \int_{0}^{1-e}\left(1-e-c-\int_{c+e}^{1}(1-q(m, c) B(b ; m, c))^{m} d b\right) d F(c)-(m+1) r^{*}+r^{*} \\
= & \int_{0}^{1-e}\left(1-e-c-\int_{c+e}^{1}(1-q(m, c) B(b ; m, c))^{m}(1-q(m, c) B(b ; m, c)) d b\right) d F(c) \\
& -(m+1) r^{*} \\
= & \Pi\left(m, r^{*}, m+1\right)
\end{aligned}
$$

together with $\partial_{r} \pi\left(m, r^{*}(m)\right)=-m>-(m+1)=\partial_{r} \Pi\left(m, r^{*}, m+1\right)$.

2) Suppose $\pi\left(m, r^{*}(m)\right) \geq \Pi\left(m, r^{*}(m), k\right)$ for all $k \in\{m+2, \ldots, K-1\}$, $K \leq n$. As we show, this implies that it also holds for $k=K$.

Obviously,

$$
\begin{aligned}
& \Pi\left(m, r^{*}(m), K\right) \\
= & \int_{0}^{1-e}\left(1-e-c-\int_{c+e}^{1}(1-q(m, c) B(b ; m, c))^{K} d b\right) d F(c)-K r^{*}(m) \\
= & \int_{0}^{1-e}\left(1-e-c-\int_{c+e}^{1}(1-q(m, c) B(b ; m, c))^{K-1} d b\right) d F(c)-(K-1) r^{*}(m) \\
& +\int_{0}^{1-e} \int_{c+e}^{1}\left((1-q(m, c) B(b ; m, c))^{K-1}-(1-q(m, c) B(b ; m, c))^{K}\right) d b d F(c)-r^{*}(m) \\
= & \Pi\left(m, r^{*}(m), K-1\right)+\int_{0}^{1-e} \int_{c+e}^{1}\left((1-q(m, c) B(b ; m, c))^{K-1} q(m, c) B(b ; m, c)\right) d b d F(c)-r^{*}(m) .
\end{aligned}
$$

Notice that

$$
\begin{aligned}
& \int_{0}^{1-e} \int_{c+e}^{1}\left((1-q(m, c) B(b ; m, c))^{K-1} q(m, c) B(b ; m, c)\right) d b d F(c) \\
& <\int_{0}^{1-e} \int_{c+e}^{1}\left((1-q(m, c) B(b ; m, c))^{m} q(m, c) B(b ; m, c)\right) d b d F(c) \\
& \quad=r^{*}(m)
\end{aligned}
$$

Therefore, $\Pi\left(m, r^{*}(m), K\right)<\Pi\left(m, r^{*}(m), K-1\right)$, as asserted.

\section{Optimal reward for bidding and size of the short-list}

We now show in two steps that it is optimal for the procurer to implement $m=2$ by offering a financial reward for short-listed bidders equal to $r=r^{*}(2)$.

In a first step we ignore the downward deviation conditions.

Lemma 3. The optimal mechanism $(m, r)$ that satisfies the upward-deviation constraints (3) is $(m, r)=\left(2, r^{*}(2)\right)$, with $r^{*}(2)=\int_{0}^{1-e} \int_{c+e}^{1}\left(\frac{e}{b-c}\right)^{2}\left(1-\frac{e}{b-c}\right) d b d F(c)$.

Proof. We have already shown that for given $m$, the reward $r^{*}(m)$ is the leastcost reward that prevents upward deviations. We now show that $\pi\left(m, r^{*}(m)\right)$ is decreasing in $m$; therefore, if one ignores downward constraints, $(m, r)=$ $\left(2, r^{*}(2)\right)$ is optimal for the procurer. 
Using Lemmas 1 and 2 together with bidders' equilibrium strategy (1), and defining $K(q, m):=(m-1) q(m, c)\left(1+\frac{m}{2} q(m, c)\right)$ one obtains

$$
\begin{aligned}
\pi\left(m, r^{*}(m)\right)= & \int_{0}^{1-e}\left(1-c-e-\int_{c+e}^{1}(1-q(m, c) B(b ; m, c))^{m} d b\right. \\
& \left.-m \int_{c+e}^{1}\left((1-q(m, c) B(b ; m, c))^{m}-(1-q(m, c) B(b ; m, c))^{m+1}\right) d b\right) d F(c) \\
= & \int_{0}^{1-e}\left(1-c-e-e(m-1) q(m, c)\left(1+\frac{m}{2} q(m, c)\right)\right) d F(c) \\
= & \int_{0}^{1-e}(1-c-e) d F(c)-e \int_{0}^{1-e} K(q, m) d F(c) .
\end{aligned}
$$

After a bit of rearranging one finds

$y(q):=\frac{\partial K(q, m)}{\partial m}=q(m)\left(1+\left(m-\frac{1}{2}\right) q(m)\right)+(1+m q(m))(1-q(m)) \log (1-q(m))$.

We show that $y(q)>0, \forall q \in(0,1)$.

We have

$y^{\prime}(q)=(m-1) q+(m-1-2 m q) \log (1-q), \quad y^{\prime \prime}(q)=\frac{(m+1) q}{1-q}-2 m \log (1-q)$.

Evidently, $y^{\prime}(0)=0$, and $y^{\prime \prime}(q)>0, \forall q \in(0,1)$ and therefore, $y^{\prime}(q)>0$, $\forall q \in(0,1)$. Again, because $y(0)=0$, it follows that $y(q)>0, \forall q \in(0,1)$. Hence, we conclude that $\pi\left(m, r^{*}(m)\right)$ is decreasing in $m, \forall q \in(0,1)$, and thus $\left(2, r^{*}(2)\right)$ is the optimal mechanism that satisfies the upward-deviation constraint.

Proposition 1. The optimal credible mechanism is $(m, r)=\left(2, r^{*}(2)\right)$. This mechanism implements the size of the short-list that is optimal under full commitment.

Proof. In Lemma 3 we have already shown that $(m, r)=\left(2, r^{*}(2)\right)$ is optimal if one ignores the downward deviations constraints. We now show that the omitted downward constraints are not binding. For this purpose, suppose $(m, r)=\left(2, r^{*}(2)\right)$ is announced. We show that the procurer cannot benefit from deviating by inviting only one bidder

$$
\pi\left(2, r^{*}(2)\right)>\Pi\left(2, r^{*}(2), 1\right) .
$$

Of course, it is not in the interest of the procurer to invite no bidder.

By Lemma 1, constraint (5) is equivalent to

$$
\begin{aligned}
r^{*}(2) & <\int_{0}^{1-e} \int_{c+e}^{1}(1-q(2, c) B(b ; 2, c)) q(2, c) B(b ; 2, c) d b d F(c) \\
& =\int_{0}^{1-e} \int_{c+e}^{1}\left(\frac{e}{b-c}\right)\left(1-\frac{e}{b-c}\right) d b d F(c) .
\end{aligned}
$$

We have $r^{*}(2)=\int_{0}^{1-e} \int_{c+e}^{1}\left(\frac{e}{b-c}\right)^{2}\left(1-\frac{e}{b-c}\right) d b d F(c)$, and hence constraint (6) is satisfied, which completes the proof. 


\section{Is it worth it?}

Rewarding short-listed bidders is costly. This raises the question: is using rewards for short-listed bidders as commitment device worth its cost? Of course, the answer depends on the parameters, in particular the number of potential contractors, $n$, and the cost of bidding $e$.

If the procurer does not offer that reward, bidders anticipate that he will invite all $n$ potential bidders. This gives rise to a most unfavorable probability distribution of the equilibrium price, which becomes less favorable, in the strong sense of first-order stochastic dominance, as $n$ is increased. Therefore, the benefit of using rewards is strictly increasing in $n$.

For each given level of the cost of bidding $e$, there is a minimal size of the pool of potential bidders, denoted by $n^{*}$, for which the reward is worth its cost, i.e., for which $\pi\left(2, r^{*}(2)\right) \geq \pi(n, 0)$. That number, $n^{*}$, is increasing in $e$. Therefore, as $e$ is increased, the thus reduced profitability of the reward scheme must be compensated by a larger minimum number of potential bidders.

The relationship between $n^{*}$ and $e$ is illustrated in Table 5, which assumes that the performance cost $c$ is uniformly distributed.

\begin{tabular}{l|rrrrrrr}
\hline $\begin{array}{l}\text { Minimal \# } \\
\text { of bidders }\end{array}$ & 0.05 & 0.075 & 0.1 & 0.125 & 0.15 & 0.175 & 0.2 \\
\hline$n^{*}$ & 4 & 6 & 7 & 9 & 11 & 17 & 33 \\
\hline
\end{tabular}

Table 1: Relationship between $n^{*}$ and the cost of bidding $e$ when $F(c)=c$

\section{Appendix}

Here we prove Lemma 1.

Proof. The c.d.f of the winning bid $b$, conditional on receiving $k$ bids, is equal to $1-(1-B(b ; m, c))^{k}$, and the expected profit of the procurer is

$$
\begin{aligned}
\Pi(m, r, a)= & \int_{0}^{1-e}\left(1-(1-q(m, c))^{a}\right. \\
& \left.-\sum_{k=1}^{a} C_{k}^{a} q(m, c)^{k}(1-q(m, c))^{a-k} \int_{c+e}^{1} b d\left(1-(1-B(b ; m, c))^{k}\right)\right) d F(c)-a r \\
= & \int_{0}^{1-e}\left(1-(1-q(m, c))^{a}-\sum_{k=1}^{a} C_{k}^{a} q(m, c)^{k}(1-q(m, c))^{a-k}(c+e)\right. \\
& \left.-\sum_{k=1}^{a} C_{k}^{a} q(m, c)^{k}(1-q)^{a-k} \int_{c+e}^{1}(1-B(b ; m, c))^{k} d x\right) d F(c)-a r .
\end{aligned}
$$

By a well-known result, $C_{k=0}^{n} x^{k} y^{n-k}=(x-y)^{n}, \forall x, y \in \mathbb{R}$, and $\forall k, n \in \mathbb{N}$. 
Therefore,

$$
\begin{aligned}
\Pi(m, r, a)= & \int_{0}^{1-e}\left(1-(1-q(m, c))^{a}-\left(1-(1-q(m, c))^{a}\right)(c+e)\right. \\
& \left.-\int_{c+e}^{1}\left(\sum_{k=1}^{a} C_{k}^{a}(1-q(m, c))^{a-k}(q(m, c)-q(m, c) B(b ; m, c))^{k}\right) d b\right) d F(c)-a r \\
= & \int_{0}^{1-e}\left(\left(1-(1-q(m, c))^{a}\right)(1-c-e)\right. \\
& \left.-\int_{c+e}^{1}\left((1-q(m, c) B(b ; m, c))^{a}-(1-q(m, c))^{a}\right) d b\right) d F(c)-a r \\
= & \int_{0}^{1-e}\left(1-c-e-\int_{c+e}^{1}(1-q(m, c) B(b ; m, c))^{a} d b\right) d F(c)-a r
\end{aligned}
$$

\section{References}

Fan, C., Wolfstetter, E., 2008. Procurement with costly bidding, optimal shortlisting, and rebates. Economics Letters 98, 327-334.

Lang, K., Rosenthal, R. W., 1991. The contractors' game. RAND Journal of Economics 22, 329-338.

Gal, S., Landsberger, M., Nemirovski, A., 2007. Participation in auctions. Games and Economic Behavior 60, 75-103.

Kaplan, T., Sela, A., 2006. Second price auctions with private entry costs. Tech. rep. Ben-Gurion University.

Tan, G., 1992. Entry and R \& D in procurement contracting. Journal of Economic Theory 22, 41-60. 